\title{
ANGIOSPERMAS UTILIZADAS NA ARBORIZAÇÃO DO CAMPUS II DA UNIFEOB, SÃO JOÃO DA BOA VISTA, SP ${ }^{1}$
}

\author{
Gabriel Augusto Martins de Melo $^{2}$, Eliana Pereira Chagas $^{3}$ \\ (recebido em 03.04.2008 e aceito para publicação em 01.12.2008)
}

\begin{abstract}
RESUMO
O campus II da Unifeob na cidade de São João da Boa Vista (SP) possui $120.000 \mathrm{~m}^{2}$ com a presença de várias áreas verdes. Foram levantadas todas as angiospermas arbóreas cujo CAP (circunferência do tronco à altura do peito) fosse igual ou superior a 60,0cm. Foram encontradas 231 árvores distribuídas em 15 famílias e 28 espécies, sendo 19 nativas e 9 introduzidas. A família mais representativa foi Leguminosae-Caesalpinoideae sendo que sibipiruna (Caesalpinia pelthophoroides Benth.) a espécie mais abundante, com 53 indivíduos. A área do campus apresentou diferenças entre as espécies encontradas em cada área. Por exemplo, o estacionamento é arborizado principalmente com acácias-australianas (Acacia mangiun Willd), uma espécie exótica, enquanto o Hovet possuía vários ipês-roxos (Tabebuia heptaphylla (Vell.) Toledo. A presença de áreas verdes em ambientes urbanos é de extrema importância, considerando-se fatores como a preservação da biodiversidade, especialmente quando se investe em espécies nativas. Planos de manejo são necessários para intensificar a preservação dessas áreas.
\end{abstract}

Palavras chave: Arborização, espécies arbóreas, levantamento

\footnotetext{
1 Nota técnica formulada a partir da monografia de graduação de Gabriel Augusto Martins de Melo, orientado pela Profa Dra. Eliana Pereira Chagas;

${ }^{2}$ Graduação em Ciências Biológicas - Licenciatura, UNIFEOB - Centro Universitário da Fundação de Ensino Octávio Bastos, Campus II Campus II, Endereço: Av. Octávio da Silva Bastos, s/ $\mathrm{n}^{\circ}$ - Pq. dos Jequitibás, 13874-422, São João da Boa Vista - SP, <gammelo@yahoo.com.br>;

3 Doutora, Professora de Botânica da UNIFEOB - Centro Universitário da Fundação de Ensino Octávio Bastos, Campus II Campus II - Av. Octávio da Silva Bastos, s/ nº - Pq. dos Jequitibás, 13874-422, São João da Boa Vista - SP, <eliana.chagas@terra.com.br>.
} 


\title{
ANGIOSPERMS USED ON AFFORESTATION OF UNIFEOB'S CAMPUS II, SÃO JOÃO DA BOA VISTA, SP
}

\begin{abstract}
The campus II of the Unifeob (São João da Boa Vista - SP - Brazil) occupies around $120.000 \mathrm{~m}^{2}$ and has many green areas. A census of the arboreal vegetation (CBH circunference at breast heigh) $\geq 60,0 \mathrm{~cm}$ ) was carried out in which 231 individuals were recorded. Twenty eight species were identifieds, distributed in 15 families, being 19 native species and 9 introduced ones. The familie with the highest richness was Leguminosae-Caesalpinoideae, being Caesalpinia pelthophoroides Benth. (sibipiruna) made up the abundant specie (53 individuals). The area of campus II displays some differences about the species by area, e.g. the Parking had only Acacia mangium Willd, an introduced specie, while the Hovet had Tabebuia heptaphylla (Vell.) Toledo a lot. The presence of plants in urban areas is very important considering the preservation of the biodiversity mainly in respect native species. Management projects are needed to intensify these preserved areas.
\end{abstract}

Key words: Arborization, trees species, survey 


\section{INTRODUÇÃO}

A presença de árvores em locais de acesso ao público nem sempre representou para a cultura ocidental uma prioridade. Cidades históricas brasileiras, assim como grandes cidades históricas da Europa não inseriam em sua arquitetura árvores nas ruas ou jardins, assim como o ambiente árido e com pedras nuas eram preferíveis dentro dos castelos europeus durante a Idade Média (SALATINO, 2001). Já a cultura oriental traz uma outra visão do ambiente arborizado, desde os míticos jardins suspensos da Babilônia até os jardins naturalistas chineses (LOBODA \& DE ANGELIS, 2005). Muito da cultura religiosa também influencia na questão da arborização. Enquanto a cultura cristã mantinha seus templos desérticos, as culturas orientais colocavam o sagrado muito ligado à natureza, como fonte de meditação e inspiração (SALATINO, 2001).

Os trabalhos de conscientização sobre a importância da preservação das áreas verdes têm sido cada vez mais realizados. MONICO (2001) promoveu uma campanha de conscientização dos moradores da cidade de Piracicaba (SP) pela manutenção das árvores das ruas da cidade. ANDRADE (2002) também relata que a diversidade de árvores das ruas de Campos do Jordão (SP) é relativamente baixa, sendo compensada pelo grande número de jardins particulares.

Alguns trabalhos vêm mostrando a importância do conhecimento das árvores plantadas em zonas urbanas (MONICO, 2001; ROCHA et al., 2004; MACHADO et al., 2006; SILVA et al., 2007;), levando em consideração que dados, como indivíduos por espécies, podem ser importantes para o planejamento da arborização urbana (AGOSTINI \& SAZIMA, 2003) assim como para ambientes universitários (PAIVA et al., 2004; KURIHARA et al., 2005).

KURIHARA et al. (2005) destacam a importância de um plano de manejo para a conservação da diversidade de espécies encontradas dentro dos campi universitário, reforçando a valorização das espécies nativas. Assim como PAIVA et al., (2004) indicam que a presença das árvores nos canteiros nas universidades, além de embelezar o ambiente, gera atividades e aulas práticas.

Este trabalho teve como objetivos amostrar e identificar as espécies arbóreas de angiospermas do campus II da UNIFEOB (Centro Universitário da Fundação de Ensino Octávio Bastos, São João da Boa Vista - SP). 


\section{MATERIAL E MÉTODOS}

O Centro Universitário da Fundação de Ensino Octávio Bastos, UNIFEOB, conta com dois campi universitários, um localizado no centro da cidade de São João da Boa Vista (SP), o campus I, e outro na região mais periférica da mesma, o campus II.

O Campus II está aproximadamente nos pontos 2158'02"S e 4646'24'W, no município de São João da Boa Vista, no estado de São Paulo, próximo à divisa com o estado de Minas Gerais. O espaço é dividido entre laboratórios e salas de aula, um amplo estacionamento, a quadra poliesportiva, a quadra de peteca, a central de cópias, a biblioteca setorial, o viveiro e o complexo do hospital veterinário dentro dos $120.000 \mathrm{~m}^{2}$ que o campus ocupa (UNIFEOB, 2007).

O levantamento ocorreu no ano de 2007 no período de fevereiro a novembro. Para organizar melhor e fazer o levantamento, o campus II foi dividido em nove blocos; 1) Guarita; 2) Bosque I; 3) Estacionamento; 4) Viveiro; 5) Xerox e bosque II; 6) Laboratórios; 7) Biblioteca; 8) Cantina e 9) Hospital Veterinário - Hovet (Figura 1).

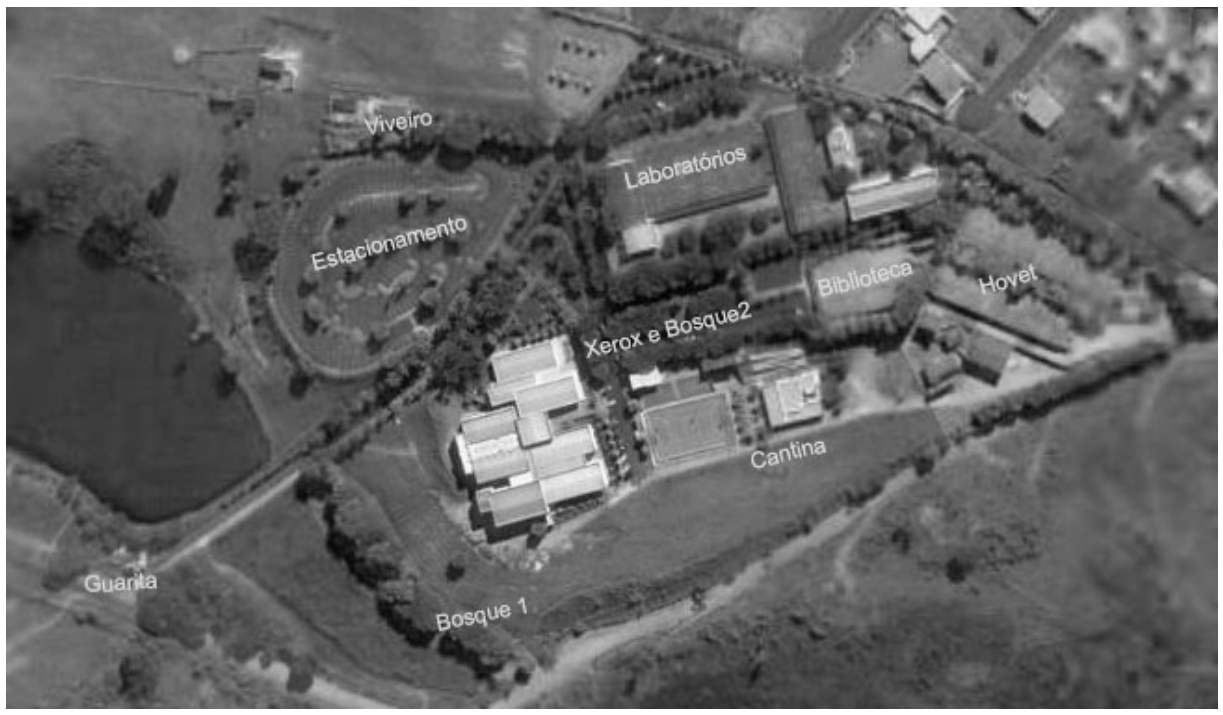

Figura 1. Campus II da Unifeob, São João da Boa Vista (SP). Foto aérea destacando as nove áreas onde foram realizados os levantamentos. Fonte: GOOGLE MAPS, 2007.

Após observação prévia definiu-se que seriam levantadas e identificadas as espécies de angiospermas arbóreas que apresentassem a circunferência à altura do peito (CAP) acima de $60,0 \mathrm{~cm}$, assim identificando apenas as plantas já adultas. Para as medições foi utilizada fita métrica. Ao encontrar espécimes onde ocorreu ramificação abaixo de 1,5 $\mathrm{m}$ de altura, foi considerado a circunferência da região mais próxima abaixo da ramificação, ou a maior 
circunferência de um dos ramos. Quando estes não apresentaram o valor estipulado, ou eram muito próximos e delgados, foi considerada a medida dos ramos juntos, como se fosse um caule conjunto.

Para a identificação das espécies foram utilizadas bibliografias específicas (LORENZI, 2002a; LORENZI, 2002b; LORENZI et al., 2003) e o acervo do herbário da UNIFEOB.

\section{RESULTADOS E DISCUSSÕES}

No estudo realizado, foram encontradas 231 angiospermas arbóreas, distribuídas em 16 famílias e 28 espécies. Não foi possível identificar 6 espécimes, pela falta de material reprodutivo e/ou outras características que tornassem possível sua identificação .

Leguminosae-Caesalpinoideae foi a família mais representativa com 7 (25\%) espécies, seguida de Leguminosae-Mimosoidae com 5 (18\%)(Figura 2).

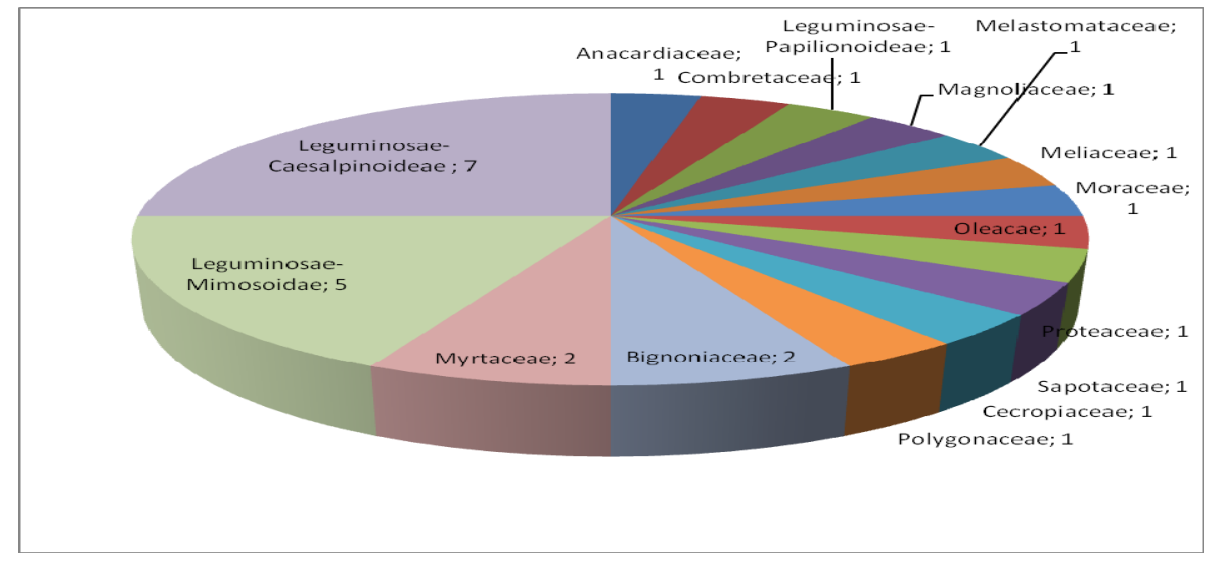

Figura 2. Distribuição das famílias botânicas por número de espécies encontradas no campus II da Unifeob, São João da Boa Vista - SP.

Dentro de todas as espécies, 19 eram nativas e 9 eram introduzidas, ou exóticas, (Tabela 1).

Dentre as espécies encontradas, aquela que mais apresentou indivíduos foi a sibipiruna, (Caesalpinia peltophoroides Benth), com 53 árvores (22,4\%). A segunda espécie mais representativa foi a Acácia-australiana (Acacia mangium Willd), com 21 indivíduos (9,4\%). Grevílea (Grevillea robusta A. Cunn. ex R.), com 20 indivíduos (8,6\%), foi a terceira mais representativa (Tabela 1 ). 
Tabela 1. Levantamento das espécies arbóreas do campus II da Unifeob.

\begin{tabular}{|c|c|c|c|c|c|c|c|}
\hline Espécie & Familia & Nome vulgar & Folha & IPA & Origem & $\begin{array}{l}\text { PAP } \\
(\mathrm{cm})\end{array}$ & $\mathrm{n}$ \\
\hline Acacia mangium Willd & Leguminosae-Mimosoidae & $\begin{array}{l}\text { acácia- } \\
\text { australiana }\end{array}$ & Semidecídua & Sim & Austrália & 79,5 & 22 \\
\hline Albizia hasslerii (Chodat) Burr & Leguminosae-Mimosoidae & farinha-seca & Decídua & * & Brasil & 78,7 & 3 \\
\hline Albizia polycephala (Benth.) Killp. & Leguminosae-Mimosoidae & angico branco & Semidecídua & $\operatorname{Sim}$ & Brasil & 215,0 & 1 \\
\hline Bauhinia variegata $\mathrm{L}$. & Leguminosae-Caesalpinoideae & pata-de-vaca & Semidecídua & Sim & Índia & 87,0 & 2 \\
\hline Caesalpinia ferrea Mart & Leguminosae-Caesalpinoideae & pau-ferro & Semidecídua & $\operatorname{Sim}$ & Brasil & 87,0 & 6 \\
\hline Caesalpinia peltophoroides Benth. & Leguminosae-Caesalpinoideae & Sibipiruna & Semidecídua & $\operatorname{Sim}$ & Brasil & 83,5 & 52 \\
\hline Cecropia sp. & Cecropiaceae & Embaúba & * & * & Brasil & 62,0 & 3 \\
\hline Cedrela fissilis Vell. & Meliaceae & Cedro & Decídua & $\operatorname{Sim}$ & Brasil & 68,3 & 3 \\
\hline Delonix regia (Boj. ex Hook.) Raf. & Leguminosae-Caesalpinoideae & falso-flamboyant & Decídua & $\operatorname{Sim}$ & Brasil & 75,7 & 3 \\
\hline Enterolobium contortisiliquum (Vell.) Morang. & Leguminosae-Mimosoidae & orelha-de-negra & Decídua & Não & Brasil & 108,0 & 1 \\
\hline Eugenia involucrata D.C. & Myrtaceae & $\begin{array}{l}\text { cerejeira-do- } \\
\text { mato }\end{array}$ & Decídua & Sim & Brasil & 72,6 & 14 \\
\hline Ficus benjamina L. & Moraceae & Figueira & Perene & Não & $\begin{array}{l}\text { Ásia/ } \\
\text { Oceania }\end{array}$ & 130,0 & 1 \\
\hline
\end{tabular}


Tabela 1. Continuação...

Grevillea robusta A. Cunn. ex R. Br

Hymenaea courbaril L.

Inga vera Willd.

Ligustrum lucidum W. T. Aiton

Lonchocarpus guilherminiana (Tul.) Malme

Machaerium nyctitans (Vell.) Benth.

Mangifera indica L.

Michelia champaca L.

Peltophorum dubium (Spreng.) Taub

Pouteria sp.

Syzygium cumini (L.) Skeels

Tabebuia alba(Cham.) Sandw

Tabebuia heptaphylla (Vell.) Toledo

\begin{tabular}{|c|c|c|c|c|c|}
\hline Proteaceae & Grevilea & Decídua & Sim & Austrália & 104,1 \\
\hline Leguminosae-Caesalpinoideae & Jatobá & Semidecídua & Não & Brasil & 70,0 \\
\hline Leguminosae-Mimosoidae & Ingá & Semidecídua & Sim & Brasil & 102,0 \\
\hline Oleaceae & Ligustro & Perene & Sim & China & 60,7 \\
\hline Leguminosae-Caesalpinoideae & pau-cigarra & Semidecídua & Sim & Brasil & 105,0 \\
\hline Leguminosae-Papilionoideae & $\begin{array}{l}\text { jacarandá-bico- } \\
\text { de-pato }\end{array}$ & Semidecídua & Sim & Brasil & 210,0 \\
\hline Anacardiaceae & Manga & Perene & Sim & Índia & 79,0 \\
\hline Magnoliaceae & $\begin{array}{l}\text { magnólia- } \\
\text { amarela }\end{array}$ & Perene & Sim & $\begin{array}{l}\text { Índia/Him } \\
\text { alaia }\end{array}$ & 72,3 \\
\hline Leguminosae-Caesalpinoideae & Canafístula & Decídua & Sim & Brasil & 91,2 \\
\hline Sapotaceae & Abiu & Semidecídua & Sim & Brasil & 70,0 \\
\hline Myrtaceae & Jambolão & Perene & Sim & Índia & 72,0 \\
\hline Bignoniaceae & ipê-amarelo & Caduca & Sim & Brasil & 60,0 \\
\hline Bignoniaceae & ipê-roxo & Caduca & Sim & Brasil & 78,6 \\
\hline
\end{tabular}

Tabela 1. Continuação... 


\begin{tabular}{|c|c|c|c|c|c|c|c|}
\hline Terminalia catappa L & Combretaceae & chapéu-de-sol & Caduca & Sim & $\begin{array}{l}\text { Madagas- } \\
\text { car }\end{array}$ & 77,0 & 11 \\
\hline Tibouchina granulosa Cong. & Melastomaceae & Quaresmeira & Perene & Sim & Brasil & 72,8 & 4 \\
\hline Triplaris americana L. & Polygonaceae & pau-formiga & Perene & Não & Brasil & 65,0 & 1 \\
\hline Não identificadas & * & * & * & * & * & * & 6 \\
\hline
\end{tabular}

CAP = circunferência à altura do peito $(\mathrm{cm}), \mathrm{n}=$ número total de indivíduos encontrados no campus II. IPA = Indicada Para Arborização em qualquer área urbana, segundo LORENZI (2002ab), LORENZI (2003) e PAIVA et al. (2004). *informação não disponível.

$\mathrm{CBH}=$ Circunference at breast height $(\mathrm{cm}), \mathrm{n}=$ total individuals found at campus II. IPA = indicated to arborization in urban areas, according to Lorenzi (2002a,b), Lorenzi et al. (2003) and Paiva et al. (2004). * = no information 
Mesmo com número de indivíduos e espécies amostrados podendo ter sofrido influência da metodologia usada, pois ao amostrar apenas indivíduos considerados adultos restringiu-se a amostragem, pois muitos não foram selecionados dado o CAP ser inferior ao estabelecido sugerindo que há a presença de indivíduos jovens.

A distribuição de espécies pelo campus II pôde ser considerada desigual, com áreas com maior diversidade, como por exemplo o Hovet, com 9 espécies diferentes, e outras como o Bosque I com uma menor diversidade, 2 espécies (Figura 3).

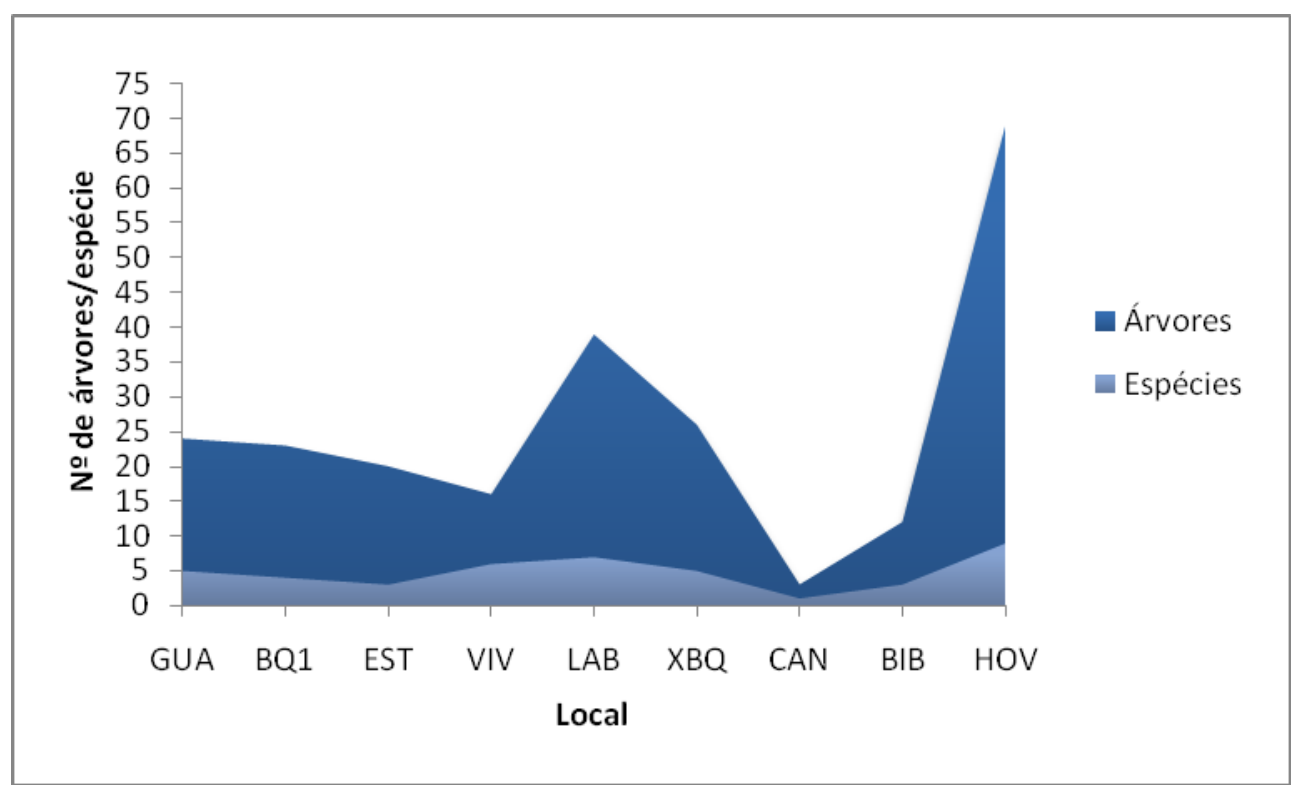

Figura 3. Distribuição de espécies e indivíduos nas nove áreas estudadas dentro do campus II da Unifeob: GUA (Guarita), BQ1 (Bosque I), EST (Estacionamento), VIV (Viveiro), LAB (Laboratórios), XBQ (Xerox e Bosque II), CAN (Cantina), BIB (Biblioteca) e HOV (Hovet).

Diferentes espécies predominam em cada área. Por exemplo, o entorno do Hovet é quase todo arborizado com uma espécie nativa, o Ipê-roxo (Tabebuia heptaphyla). Já o entorno do Viveiro é quase todo arborizado com Chapéu-de-sol (Terminalia catappa) e Magnólias (Michelia champaca); enquanto o Estacionamento é praticamente todo arborizado com Acácias australianas (Acacia mangium), uma espécie utilizada em paisagismo (INSTITUTO HÓRUS, 2008). O chamado Bosque I possui basicamente Ingás (Inga vera) e Jambolões (Syzygium cumini). Algumas espécies foram esporádicas, como por exemplo, o Jatobá (Hymenaea courbaril), encontrado no Xerox e Bosque II, e o Pau-Cigarra (Lonchocarpus guilherminiana), observado na guarita.

Em áreas como o Estacionamento onde se tem a predominância de Acáciasaustralianas (Acacia mangium), talvez sejam necessários planos de manejo, e como sugerido por CIELO FILHO E SANTIN (2002), no estudo realizado em bosques dentro da 
cidade de Campinas, que se removam gradativamente essas espécies e sejam substituídas por espécies nativas. Um exemplo de área arborizada dentro do campus com espécies nativas foi a áreas do Hovet, onde em quase todo o seu entorno foram encontrados Ipêsroxo (Tabebuia heptaphyla) e também as áreas do Bosque I com a prevalência de Jambolão (Syzygium cumuni) e Ingá (Inga vera).

A área dos Laboratórios é utilizada em parte como estacionamento e outra como área de dispersão após as aulas práticas, mostrando também uma necessidade de sombra em dias quentes. Segundo FEDRIZZI et al. (2007), áreas sombreadas em escolas, além de serem reivindicações de alunos, atuam como fator paisagístico e no despertar de uma consciência ambiental, támbém apaziguando o clima e valorizando o processo ensinoaprendizagem.

Todavia, mesmo com a predominância de sibipirunas (Caesalpinia peltophoroides) na vegetação do Campus II da Unifeob, as árvores ali encontradas também servem a propostas paisagísticas e didáticas, o que também foi verificado por PAIVA et al. (2004). Em um levantamento no canteiro central da Universidade de Lavras, estes autores observaram que as árvores ali presentes são de extrema importância para aulas práticas e os estudos provenientes destas, como coleta de sementes, proporcionando ainda um maior conhecimento das espécies existentes. Tais conhecimentos apenas reforçam a importância da arborização existente (KURIHARA et al., 2005), e quando arborizados, os campi universitários atuam como coleções vegetais, tendo fins didáticos e científicos, mostrando grande influência no ambiente urbano (COSTA, 2004).

Ambientes com vestígios de mata nativa, ou pequenas áreas verdes, como no caso do campus II, apresentam grande função ecológica, atuando na preservação da biodiversidade local (SILVA E BORTOLETO, 2005). Além das áreas verdes urbanas, é necessária a preservação dos fragmentos de matas ainda existentes (HENRIQUES, 2003), através da inserção de planos de manejo e conservação de remanescentes, mantendo vestígios de mata nativa (RINGOLIN DE SÁ et al., 2001), assegurando desse modo permanência da fauna de da flora que ainda estão nestes locais, contribuindo para que não se perca pelo menos parte dessa biodiversidade. A UNIFEOB é proprietária de uma região de mata ciliar, pertencente à Fazenda Escola (Fazenda Prata), e que segundo ARAÚJO (2004), é um importante fragmento de mata nativa. Uma ação que poderia ser adotada pela administração do campus seria restaurar e aumentar essa área na fazenda, preservando as espécies nativas, a mata e tudo que a compõe, conservando e aumentando também a fauna local, além da própria vegetação do campus II.

Como se pôde verificar, o campus II apresentou uma certa diversidade de espécies, sendo maior em alguns setores, como nos Laboratórios e na área do Hovet, e menores 
como na Cantina, no Estacionamento e no Viveiro. A presença dessas árvores tem uma importância inquestionável, tanto científica, educacional e ambiental quanto paisagística.

Assim, a preservação e o plantio de espécies nativas mantém não só todas essas características citadas, como também mantém todo um patrimônio de fauna e flora nativas, desde suas características genéticas até aquelas relacionadas às suas comunidades.

\section{REFERÊNCIAS BIBLIOGRÁFICAS}

AGOSTINI, K., SAZIMA, M. Plantas ornamentais e seus recursos para abelhas no campus da Universidade Estadual de Campinas, Estado de São Paulo, Brasil. Bragantia, Campinas, v.62, n.3. p.355-343, 2003.

ANDRADE, T. O., Inventário e análise da arborização viária da Estância Turística de Campos do Jordão, SP. Dissertação (Mestrado em agronomia) Escola Superior de Agricultura "Luiz de Queiroz", Universidade de São Paulo, Pirassununga, 2002, 112p.

ARAUJO, M. H. T. Levantamento Florístico dos Fragmentos de Mata Ciliar da Fazenda Prata, São João da Boa Vista - SP. Monografia (Graduação em Ciências Biológicas) Centro Universitário da Fundação de Ensino Octávio Bastos, São João da Boa Vista, 2004. 45p.

CIELO FILHO, R., SANTIN, D. A. Estudo florístico e fitossociológico de um fragmento florestal urbano - Bosque dos Alemães, Campinas, SP. Rev. Brasil. Bot., São Paulo, v.25, n.3. p. 291-301, 2002.

COSTA, F. P. S., Evolução urbana e da cobertura vegetal de Piracicaba - SP (19402000). Dissertação (Mestrado em Ecologia de Agroecossistemas) Escola Superior de Agricultura "Luiz de Queiroz" - USP-SP. Piracicaba, 2004. 96p.

FEDRIZZI, B., TOMASINI, S. L. V., CARDOSO, L. M.. A vegetação no pátio escolar: um estudo par a realidade de Porto Alegre - RS. $2007 \mathrm{~m}$ Disponível em <http://www.sbau.org.br/materias_sergio_toma.html> acesso em 16 de jun 2007.

GOOGLE MAPS. Mapas Brasil. Disponível em <http://maps.google.com.br/maps?utm_campaign=pt_BR\&utm_source=pt_BR-ha-latam-brbk-gm\&utm_medium=ha\&utm_term=google\%20maps> acesso em 06 de março de 2007. 
HENRIQUES, O. K., Caracterização da vegetação natural em Ribeirão Preto, SP: Bases para conservação. Dissertação (Doutorado em Biologia Comparada) Universidade de São Paulo, Ribeirão Preto, 2003. 221p.

INSTITUTO HÓRUS. Fichas Técnicas. Disponível em <http://www.institutohorus.org.br/index.php?modulo=inf_ficha_acacia_mangium> acesso em 10 nov 2008.

KURIHARA, D. L.; IMANÃ-ENCINAS, J., PAULA, J. E. Levantamento da arborização do Campus da Universidade de Brasília. Cerne, Lavras-MG, v.11 n.2, p.127-136 abr./jun., 2005

LOBODA, C. R., DE ANGELIS, B. L. D. Áreas verdes públicas urbanas: Conceitos, usos e funções. Ambiência. Guarapuava-PR. v.1 n. 1, p. 125-139. jan/jun, 2005.

LORENZI, H. Árvores Brasileiras: Manual para identificação e cultivo de plantas arbóreas nativas do Brasil. v.1 2.ed. Nova Odessa : Editora Plantarum, 2002a.

Árvores Brasileiras: Manual para identificação e cultivo de plantas arbóreas nativas do Brasil. v.2 2.ed. Nova Odessa : Editora Plantarum, 2002b.

, SOUZA, H. M., TORRES, M. A. V., BACHER, L. B. Árovres Exóticas no Brasil: madereiras, ornamentais e aromáticas. Nova Odessa : Editora Platarum, 2003.

MACHADO, R. R. B., MEUNIER, I. M. J., SILVA, J. A. A., CASTRO, A. A. J. F., Árvores nativas para a arborização de Teresina, Piauí. Rev. Soc. Bras. Arb. Urb., Piracicaba-SP, v.1, n.1, p.10-18. 2006.

MONICO, I. M. Árvores e arborização urbana na cidade de Piracicaba/SP: Um olhar sobre a questão à luz da educação ambiental. Dissertação (Mestrado em Ciências Florestais) Escola Superior de Agricultura Luiz de Queiroz, Piracicaba, 2001, 165p.

PAIVA, P. D. O.; LANDGRAF, P. R. C.; RODIGUES, T. M.; PEDROSO, D. O.; OLIVEIRA FILHO, A. T.; GAVILANES, M. L., PAIVA, R. Identificação e caracterização das espécies arbóreas do Canteiro Central da Universidade Federal de Lavras/MG. Ciênc. Agrotec. Lavras-MG, v.28, n.3, p.515-519, maio/jun., 2004. 
ROCHA, Y. T., CAVALHEIRO, F.. Aspectos históricos do Jardim Botânico de São Paulo. Rev. Bras. Bot., São Paulo, v. 24, n. 4, 2001.

ROCHA, R. T., LELES, P. S. S., OLIVEIRA NETO, S. N. Arborização de vias públicas em Nova Iguaçu, RJ: O caso dos bairros Rancho Novo e Centro. Árvore, Viçosa-MG v.28, n.4, p.599-607, 2004.

RIGOLIN DE SÁ, O.; QUEIROZ, A. C.; OLIVEIRA, T. C. T.; OLIVEIRA, K. C.; FRANÇA, N.; OLIVEIRA, J. R.; PEREIRA, K. C.; FRANCISCO, B. C.; MAZON, A. F., Levantamento das áreas verdes urbanas do município de Passos, MG. 2001 In. CONGRESSO DE ECOLOGIA DO BRASIL, AMBIENTE E SOCIEDADE. Porto Alegre, RS. Resumos... Porto Alegre - PR. p 411, 2001.

SAlatino, A, Nós e as Plantas: ontem e hoje. São Paulo : Rev. Brasil. Bot. v.24, n.4, p.483-490, 2001.

SILVA, D. F.; BORTOLETO, S. Uso de indicadores de diversidade na definição de plano de manejo da arborização viária de Águas de São Pedro - SP. Árvore, Viçosa-MG, v.29, n.6, p.973-982, 2005.

SILVA, L. M.; MOCCELLIN, R.; WEISSHEIMER, D. I.; ZBORALSKI, A. R.; FONSECA, L., RODIGHIERO, D. A. Inventário e sugestões para arborização em via pública de Pato Branco/PR. Rev. Soc. Bras. Arb. Urb., Pirassununga-SP. v.2 n.1, p.100-108, 2007.

UNIFEOB. Campus II. Disponível em

$<$ http://www.unifeob.edu.br/novo/cursos/cbiologicas/index_campus.asp> acesso em 05 de janeiro 2007. 